\title{
Crippling bone deformities and a palpable neck lump; rare presentation of a giant parathyroid adenoma
}

\author{
Zain Ul Abideen ${ }^{1}$, Adnan Arif ${ }^{2}$, Abdullah Abbasi $^{3}$, Chaudhary Muhammad Junaid Nazar ${ }^{*}$, Fizza Aroj ${ }^{5}$
}

\begin{abstract}
The most common cause of primary hyperparathyroidism is a solitary parathyroid adenoma. The different manifestations of the disease are due to excess parathyroid hormone (PTH) secretion. "Giant parathyroid adenomas" weigh more than $2 \mathrm{~g}$ and are rarely evident in clinical examination. We present a young man who presented with severe bone deformities and a palpable neck lump. After surgery, a giant parathyroid adenoma was removed that weighed $33 \mathrm{~g}$. Adenomas weighing more then $30 \mathrm{~g}$ are rare and presentation as a neck lump and severe bone disease has seldom been described. In patients presenting with a palpable neck lump, severe bone disease and renal calculi giant parathyroid adenomas should be considered as a possible culprit. Keywords: Primary hyperparathyroidism, Giant parathyroid adenoma, Osteitis fibrosa cystica, Parathormone Please cite this paper as: Ul Abideen Z, Arif A, Abbasi A, Nazar CMJ. Crippling bone deformities and a palpable neck lump; rare presentation of a giant parathyroid adenoma. J Parathyr Dis. 2017;5(2):38-41. DOI: 10.15171/jpd.2017.03.

Copyright ( $) 2017$ The Author(s); Published by Nickan Research Institute. This is an open-access article distributed under the terms of the Creative Commons Attribution License, which permits unrestricted use, distribution, and reproduction in any medium, provided the original work is properly cited.
\end{abstract}

\section{Introduction}

The incidence of primary hyperparathyroidism is 25 cases per 100000 per year (1). The most common cause in $85 \%$ of patients is a solitary parathyroid adenoma. Multiple adenomas, parathyroid hyperplasia and carcinoma account for the remaining cases (2). Parathyroid adenomas generally remain small in size and their average weight is between $70 \mathrm{mg}$ to $1 \mathrm{gm}$ (3). Those weighing more than 2 g are termed "giant parathyroid adenomas" (4). Interestingly, past studies revealed a direct relationship between gland weight and functional activity, as well as risk of post operative hypocalcaemia after parathyroidectomy $(5,6)$. Generally, giant parathyroid adenomas do not produce severe symptomatic disease (7).

These tumours are not appreciable on clinical examination of the neck. Imaging modalities used for localizing the tumours are non-invasive. Ultrasonography (USG) of the neck, parathyroid scintigraphy and magnetic resonance imaging (MRI) of the neck may be used if surgical treatment is being planned (2).

We present the interesting case of a young man with severe recurrent bone and renal disease who presented with an enlarging neck lump. We were surprised to come across a giant parathyroid adenoma during surgery that was the culprit behind these features and was producing the swelling in the neck. This atypical scenario, of a giant parathyroid adenoma, manifesting as a neck lump and producing severe bone disease is a rare occurrence and deserves a special mention.

\section{Case Report}

A-38-year old male labourer presented with progressively severe pain in his limbs and abdomen for the past 4 months. He had severe limb deformities including bowing of his lower limbs and kyphosis which had made him bed ridden. He also had severe epigastric distress which was diagnosed as acid peptic disease a few months ago. In addition he also had polyuria, polydipsia and weight loss. He described a small swelling in the neck for the last 6 months which had grown in size over the last 3 months. On examination, there was a palpable swelling in the lower right part of the neck which moved with swallowing. It was ovoid in shape, firm in consistency, non-tender with normal overlying skin and smooth surface, and had a size of $4 \times 4 \times 3 \mathrm{~cm}$.

The thyroid hormone levels were normal. USG of the neck revealed a hypo echoic region in the inferior aspect of the right lobe of the thyroid gland, anatomically distinct from the gland.

Serum calcium level was $15.1 \mathrm{mg} / \mathrm{dL}$. Serum parathyroid hormone (PTH) was $2500 \mathrm{ng} / \mathrm{dL}$ (normal 12-75 ng/dL) and alkaline phosphatase (ALP) was 3165 IU/L (44-147 $\mathrm{IU} / \mathrm{L})$. Serum phosphorus was $1.6 \mathrm{mg} / \mathrm{dL}(2.4-4.1 \mathrm{mg} / \mathrm{dL})$. Serum urea was $52 \mathrm{mg} / \mathrm{dL}(20-40 \mathrm{mg} / \mathrm{dL})$ and serum creatinine was $2.0 \mathrm{mg} / \mathrm{dL}(0.5-1.5 \mathrm{mg} / \mathrm{dL})$

$\mathrm{X}$-rays of the long bones showed severe osteopenia and 


\section{Implication for health policy/practice/research/ medical education \\ In patients presenting with a palpable neck lump, severe bone disease and renal calculi giant parathyroid adenomas should be considered as a possible culprit.}

lytic (punched out) lesions (Figure 1). X-rays and USG of the pelvis showed bilateral nephrolithiasis and severe osteopenia of the vertebral bodies (Figure 1).

Technetium sestamibi scan revealed increased radio tracer uptake at the lower pole of the right thyroid lobe (Figure 2).

MRI of the neck revealed a $3.5 \times 3 \mathrm{~cm}$ heterogeneous soft tissue intensity nodule in the posterior inferior aspect of the right thyroid lobe with anterior displacement of the thyroid suggestive of a parathyroid adenoma (Figure 3 ).

Patient underwent a neck exploration for the adenoma, which was isolated from the right retro thyroid area (Figure 4). It was then sent for histopathology which revealed typical parathyroid tissue. Weight of the gland was $33 \mathrm{~g}$ with dimensions $5 \times 4 \times 4 \mathrm{~cm}$. The PTH levels normal-

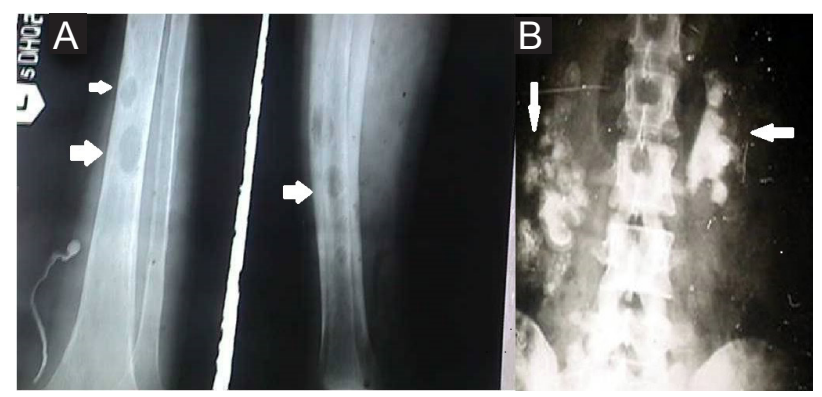

Figure 1. Panel A shows 2 views of the left lower limb. Notice the severe osteopenia, the lytic (punched out) lesions and the bowing of the bones in the second image of Panel A. Panel B is an $\mathrm{x}$-ray of the pelvis showing large bilateral renal calculi and severe osteopenia of the vertebral bodies.

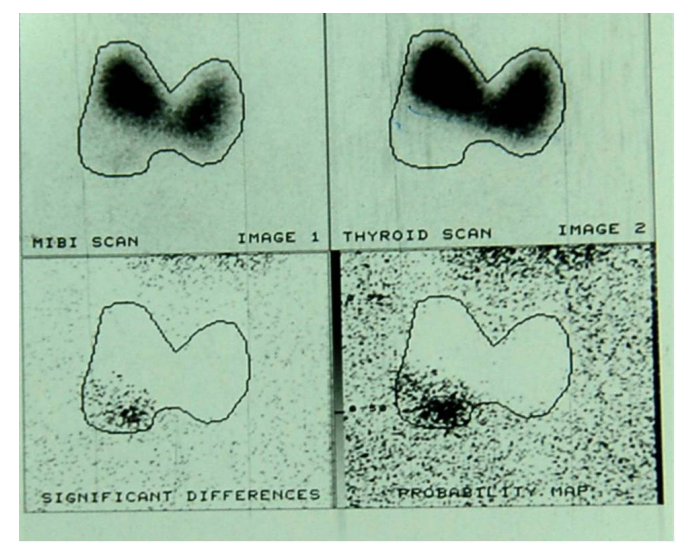

Figure 2. Technetium sestamibi scan of the thyroid gland; there is normal tracer uptake by the thyroid gland shown in the upper panels of the image. The lower panel shows an initial image showing increased uptake of the radio tracer in the lower pole of the right thyroid lobe. The delayed image shows persistent uptake of the tracer in the same area, suggestive of parathyroid adenoma. ized 36 hours after surgery. There was no episodes of hypocalcemia and no intra or post procedure complication. Our patient's symptoms resolved completely. He comes for routine follow up and is finally enjoying a healthy life.

\section{Discussion}

Giant parathyroid adenomas are an uncommon surgical disease (1). The primary mechanism for its clinical manifestations include increased production of the PTH culminating in hypercalcemia and hypophosphatemia by acting directly on the bones and kidney respectively, and on the intestines via vitamin $\mathrm{D}$.

We searched the literature on the sizes reported and found only about 25 cases reported which weighed more then 30 $\mathrm{g}$. The adenoma removed from our patient was $33 \mathrm{~g}$. A direct relationship exists between the weight of the gland and its functional activity, however, severe bone disease has seldom been described with giant adenomas. The classic bone disease in hyperparathyroidism is called osteitis fibrosa cystica. This is diagnosed by the typical lytic or punched out lesions. There is accompanying severe osteopenia in the vicinity which makes bones prone to

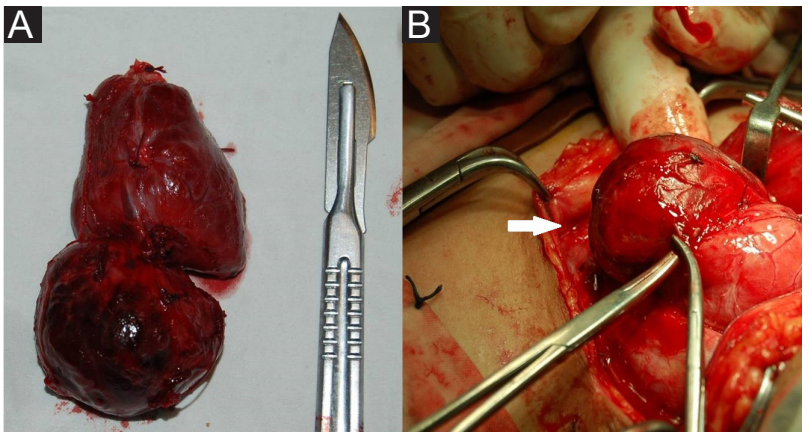

Figure 3. Magnetic resonance imaging of the neck revealed a $3.5 \times 3 \mathrm{~cm}$ heterogeneous soft tissue intensity nodule in the posteroinferior aspect of the right thyroid lobe with anterior displacement of the thyroid suggestive of a parathyroid adenoma

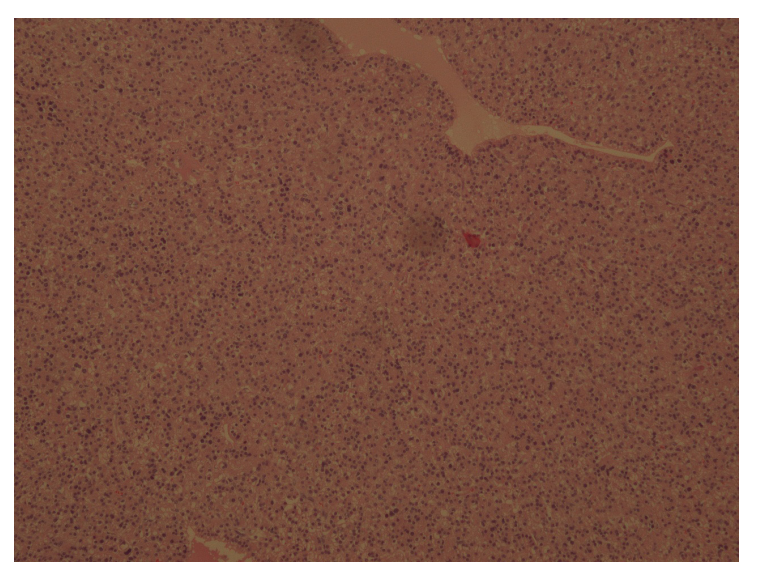

Figure 4. Post- and intraoperative views of the parathyroid adenoma. Panel A shows the large adenoma removed after the surgery. Panel B shows the adenoma after isolation during the surgery. 
fractures and bowing. Our patient had severe parathyroid bone disease too (8-11).

The second rare aspect of our case apart from the size, was the presentation of the adenoma as a palpable neck lump. Parathyroid glands lie behind the thyroid glands and are not clinically palpable even when enlarged. Only a few cases reported, exist regarding this presentation. Imaging modalities further help in identifying the cause of the neck swelling as the parathyroid gland and aided in guiding us to the position of the gland and its estimated size. Parathyroid gland localization by various imaging modalities helps in decreasing operative time and potential morbidity. This also allows for a unilateral neck exploration to decrease risk of post operative hypocalcaemia and recurrent laryngeal nerve paralysis (9-12). Parathyroid scintigraphy accurately localizes the glands in over $80 \%$ of cases (13). Our patient's adenoma was localized accurately by this modality. USG of the neck complements sestamibi scan. This is most helpful in localizing intra-thyroidal parathyroids $(9,13)$. In our patient this modality complemented sestamibi scan's findings. Computed tomography (CT) scan and MRI are less sensitive, however they may help in localizing mediastinal glands (13). The MRI scan is our patient, localized the lesion fairly well and gave us a good idea of the position of the gland pre operatively.

The most common approach to removing the adenoma is via a collar incision which also helps in examining all 4 glands for disease activity. A similar approach was used in our patient. Some investigations reported a higher risk of postoperative hypocalcemia and tetany in patients with giant adenomas. We monitored our patient stringently for tetany, however our patients did not experience tetany post-operatively.

Parathyroid adenomas produce an excess of PTH which causes hypercalcemia and hypophosphatemia. This is mediated by the PTH directly via its actions on the bones and kidney and indirectly through vitamin $\mathrm{D}$ in the intestines (2). Our patient had a typical biochemical picture of primary hyperparathyroidism corresponding to high calcium, low phosphorus and elevated PTH.

The most common presenting presentations of the disease include muscular weakness (70\%), myalgia (54\%), arthralgia (54\%), constipation (32\%) and nephrolithiasis (30\%) and polyuria (28\%). Less commonly a patient may present with symptomatic bone disease $(2 \%)$.

The extreme bone deformities in our patient were due to a disease called osteitis fibrosa cystica as the classic bone disease in primary hyperparathyroidism. It presents with bony pains, fractures and bowing of weight bearing bones (8). Radiography of the long bones typically shows lytic or punched out lesions and severe osteopenia in the vicinity. The interesting aspect of our case was the discovery of a giant adenoma weighing $33 \mathrm{~g}$ that was palpable as a neck lump and produced this severe bone disease. Normally, each gland is $3 \times 4 \times 5 \mathrm{~mm}$ size and weighs between 70 $\mathrm{mg}$ to $1 \mathrm{~g}(1,4)$. Giant adenomas weigh above $2 \mathrm{~g}$. Previous investigations revealed almost 25 case reports of giant adenomas weighing more the $30 \mathrm{~g}$. The largest adenoma removed was $145 \mathrm{~g}$ (9). Rarely, these adenomas may be bilateral (10).

Giant parathyroid adenomas usually do not produce such severe clinical disease and bone deformities, despite their large sizes and increased glandular activities. A direct relationship has been established between gland weight and its functional status (7). Only one case of giant non-functioning parathyroid adenoma has been reported (11). However, they may be associated with severe hypocalcemia post-surgery. Our patient however, did not develop hypocalcemia post-operatively.

Parathyroid gland localization by various imaging modalities helps in decreasing operative time and potential morbidity. This modality also allows for a unilateral neck exploration to decrease risk of post-operative hypocalcaemia and recurrent laryngeal nerve paralysis (12). Parathyroid scintigraphy accurately localizes the glands in over $80 \%$ of cases (13).

\section{Conclusion}

Through this case report, we concluded that giant parathyroid adenomas are an uncommonly encountered surgical disease and in very rare circumstances they may present as an enlarging neck lump and severe symptomatic bone disease.

\section{Authors' contribution}

All authors contributed equally to the manuscript.

\section{Conflicts of interest}

The authors declared no competing interests.

\section{Ethical considerations}

Ethical issues (including plagiarism, data fabrication, double publication) have been completely observed by the authors.

\section{Funding/Support}

None.

\section{References}

1. Gauger PG, Doherty GM. Parathyroid Gland. In: Townsend CM, Evers BM, Beauchamp RD, Mattox KL, eds. Sabiston Textbook of Surgery. 17th ed. Philadelphia: Saunders/ Elsevier; 2004. p. 1063-78.

2. Potts JT Jr, Jüppner H. Disease of the parathyroid gland and other hyper and hypo calcemic disorders. In: Fauci AS, Braunwald E, Isselbacher KJ, Wilson JD, Martin JB, Kasper DL, eds. Harrison's Principles of Internal Medicine. 16th ed. New York: McGraw-Hill Co; 2005. p. 2249-67.

3. Salehian MT, Namdari O, Mohammadi SS, Fazaeli Yousofabad H. Primary Hyperparathtyroidism due to a giant parathyroid adenoma: a case report. Int J Endocrinol Metab. 2009;2:101-5.

4. Meyer-Rochow GY, Sidhu SB, Delbridge LW, Grodski S,Sywak MS. ES12 Can giant parathyroid adenomas be safelyremoved by minimally invasive parathyroidectomy? ANZ J Surg. 2007;77(Suppl 1):A23.

5. Rao DS, Honasoge M, Divine GW, Phillips ER, Lee MW, Ansari MR, et al. Effect of vitamin D nutrition on 
parathyroid adenoma weight: pathogenetic and clinical implications. J Clin Endocrinol Metab. 2000;85:1054-63.

6. Mozes G, Curlee KJ, Rowland CM, Van Heerden JA, Thompson GB, Grant CS. The predictive value of laboratory findings in patients with primary hyperparathyroidism. J Am Coll Surg. 2002;194:126-30.

7. Spanheimer PM, Stoltze AJ, Howe JR, Sugg SL, Lal G, Weigel RJ. Do giant parathyroid adenomas represent a distinct clinical entity? Surgery. 2013;154:714-8.

8. Kemp AM, Bukvic M, Sturgis CD. Fine needle aspiration diagnosis of osteitis fibrosa cystic (Brown tumor of bone): a case report. Acta Cytol. 2008;52:471-4.

9. Cakmak H, Tokat AO, Karasu S, Özkan M. Giant mediastinal parathyroid adenoma. Tuberk Toraks. 2011;59:263-5.
10. Bayraktar B, Demiral G, Ozemir IA, Bayraktar O, Mehmet Yilmabasar M, Boluk S. Bilateral giant mediastinal parathyroid adenomas presented with severe hypercalcemia: report of a case. J Med Cases. 2014;5:167-70.

11. Kiverniti E, Kazi R, Rhys-Evans P, Nippah R. Airway obstruction due to giant non-parathyroid hormone producing parathyroid adenoma. J Cancer Res Ther. 2008;4:197-9.

12. Roe SM, Burns RP, Graham LD, Brock WB, Russel WL. Cost effectiveness of preoperative localization studies in primary hyperparathyroid disease. Ann Surg. 1994;219:582-6.

13. Brunicardi FC, Andersen DK, Billiar DR, Dunn DL, Hunter JG, Poilock RE. Schwartz's Manual of Surgery. 8th ed. New York: Mcgraw Hill; 2006. p. 962-74. 